\title{
FIBRING THE COMPLEMENT OF THE FENN-ROLFSEN LINK
}

\author{
ROGER FENN
}

\begin{abstract}
In this note it is shown that the complement of the singular linked spheres in four dimensions defined by Fenn and Rolfsen can be fibred by tori.

Also a symmetry between the two components is revealed which shows that the image provides an example of a Spanier-Whitehead duality. This provides an immediate proof that the $\alpha$-invariant is non zero.
\end{abstract}

\section{Introduction}

Define a link map of two spheres to be a map

$$
f: S_{1}^{p} \cup S_{1}^{q} \longrightarrow \mathbf{R}^{m}\left(\text { or } S^{m}\right)
$$

of disjoint spheres of dimension $p$ and $q$ into a Euclidean space or sphere of the same dimension $m$, such that the images of the two components are disjoint.

$$
\text { So } f\left(S_{1}^{p}\right) \cap f\left(S_{2}^{q}\right)=\phi \text {. }
$$

Let $f_{1}=f \mid S_{1}^{p}$ and $f_{2}=f \mid S_{2}^{q}$. By an abuse of notation the suffixes 1 and 2 will often be dropped when no confusion can arise.

In this paper only low dimensions will be considered: in particular the classical case $p=q=1, m=3$ and the next interesting case $p=q=2, m=4$.

For higher dimensions see $[\mathrm{K}]$ or $[\mathbf{F}, \mathrm{H}]$.

Two link maps are said to be link-homotopic if they are homotopic through link maps.

Given a link map $f$ as above there is a map

$$
\varphi: S^{p} \times S^{q} \longrightarrow S^{m-1}
$$

defined by

$$
\varphi(x, y)=\frac{f(x)-f(y)}{\|f(x)-f(y)\|}
$$

This work was written while the author was at the Centre de Recerca Matematica, Barcelona. He thanks this institution for its hospitality and support. 
The homotopy class of $\varphi$ written $\alpha(f)$, may be regarded as lying in $\pi_{p+q}\left(S^{m-1}\right)$ if $p, q \leq m-2$ and is a link homotopy invariant.

In the classical case $\alpha(f)$ is an integer and corresponds to the linking number. Moreover $\alpha(f)$ in this dimension ciassifies links up to homotopy.

As examples consider the Hopf link and the whitehead link illustrated in figure 1 .
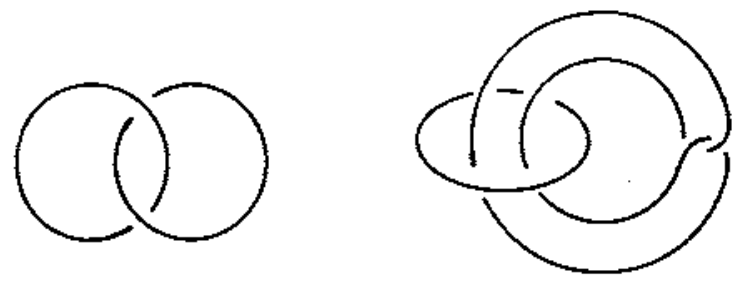

Figure 1

Hopf link

Whitehead link

The Hopf link has linking number \pm 1 and so is not null homotopic (homotopic to a pair of points).

However the Whitehead link is nullhomotopic as can easily be seen. Actually it will be shown that the Whitehead link is nullhomotopic in a rather stronger sense which depends on its symmetry.

For the case of link maps $f: S^{2} \cup S^{2} \rightarrow \mathbf{R}^{4}$ the $\alpha$-invariant lies in $\pi_{4} S^{3} \cong \mathbf{Z}_{2}$. It is known that $\alpha$ is not a complete invariant in this case, see [K].

\section{Constructing linked 2-spheres from linked circles}

A link map $f: S^{2} \cup S^{2} \rightarrow \mathrm{R}^{3}$ is said to be doubly null homotopic if each component is null homotopic in the complement of the other. For linked circles with unknotted components being doubly nullhomotopic is the same as having linking number zero although it is not often easy to see.

Figure 2 illustrates the fact that the Whitehead link is doubly null homotopic. 


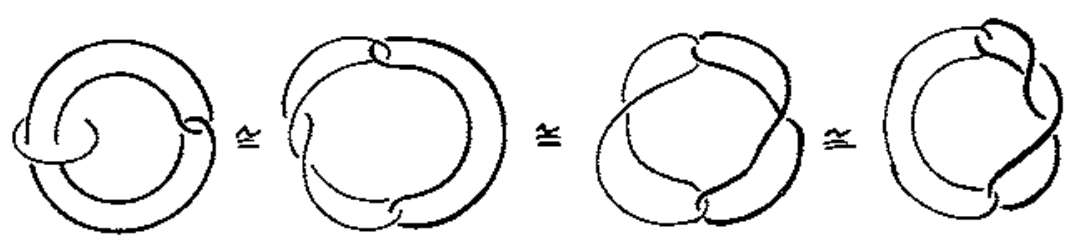

Figure 2. Symmetry of the Whitehead link

Let $f_{1 t}, f_{2 t}, 0 \leq t \leq 1$ denote the homotopies of a doubly nullhonotopic link. So $f=f_{10} \cup f_{20}$ whilst $f_{11}$ and $f_{21}$ are constant maps. Moreover $f_{1 \imath} \cup f_{20}$ and $f_{10} \cup f_{2 t}$ are link-homotopies which shrink one component while fixing the other.

Let $(x, t), x \in S^{1}$ and $-1 \leq t \leq 1$, denote cylindrical coordinate on the sphere $S^{2}$.

Define $\hat{f}: S_{1}^{2} \cup S_{2}^{2} \rightarrow \mathrm{R}^{4}$ by

$$
\begin{gathered}
\hat{f}\left(x_{1}, t\right)=\left\{\begin{array}{lr}
(1-t)\left(f_{1 t}\left(x_{1}\right), t\right), & 0 \leq t \leq 1, x_{1} \in S_{1}^{1} \\
(1+t)\left(f_{10}\left(x_{1}\right), t\right), & -1 \leq t \leq 0, x_{1} \in S_{1}^{1}
\end{array}\right. \\
\hat{f}\left(x_{2}, t\right)=\left\{\begin{array}{lr}
(1-t)\left(f_{20}\left(x_{2}\right), t\right), & 0 \leq t \leq 1, x_{2} \in S_{2}^{1} \\
(1+t)\left(f_{2-t}\left(x_{2}\right), t\right), & -1 \leq t \leq 0, x_{2} \in S_{2}^{1}
\end{array}\right.
\end{gathered}
$$

Diagramatically we can think of $\hat{f}$ as in figure 3 .

$\hat{f}_{1}$

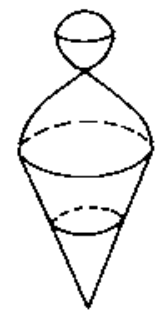

$\hat{f}_{2}$

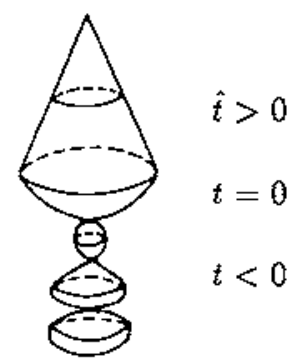

Figure 3. Constructing $\hat{f}$

The correspondence $f \rightarrow \hat{f}$ is well defined up to link homotopy because of the asphericity of knots.

If $f$ is the Whitehead link, $\hat{f}$ is the Fenn-Rolfsen link. This case is illustrated in figure 4. Sections are drawn according to approximate choices of the coordinate $t$. 

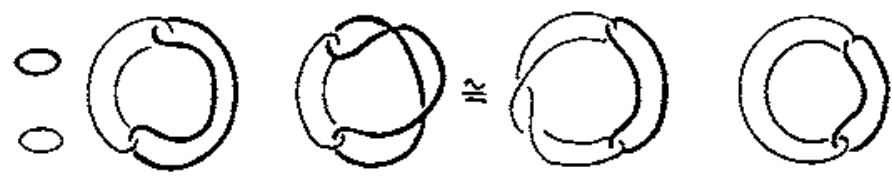

$t_{0}$

$t_{1}$

$t_{2}$

$t_{3}$

Figure 4. Sections of the Fenn-Rolfsen link

In figure 4 critical occurences happen at times $t_{0}, t_{1}, t_{2}, t_{3}$. They are described in the following list:

$$
\begin{aligned}
& t_{0}: \text { minima of } f_{1} \text { and } f_{2} \\
& t_{1}: \text { crossing point of } f_{1} \\
& t_{2}: \text { crossing point of } f_{2} \\
& t_{3}: \text { maxima of } f_{1} \text { and } f_{2}
\end{aligned}
$$

Between $t_{1}$ and $t_{2}$ is an isotopy of the Whitehead link.

\section{The double Hopf map}

Let $h: S^{3} \rightarrow S^{2}$ denote the Hopf map. If $x \in S^{2}$ then $h^{-1} x$ is an unknotted circle and for two distinct points $x, y \in S^{2} h^{-1} x, h^{-1} y$ is a Hopf link. As $x$ varies over $S^{2}$ the circles $h^{-1} x$ form the Hopf fibration of $S^{3}$.

Consider the suspension of $h ; \Sigma h: \Sigma S^{3} \rightarrow \Sigma S^{2}$.

Identify $\Sigma S^{3}$ with $S^{4}$ and $\Sigma S^{2}$ with $S^{3}$. Under the identification of $\Sigma S^{2}$ with $S^{3}$ let the suspension points lie in $h^{-1} x_{+}$and $h^{-1} x_{-}$. The composition of $\Sigma h$ with $h$ gives a map $S^{4} \stackrel{s}{\longrightarrow} S^{2}$.

$$
S^{4} \cong \Sigma S^{3} \stackrel{\Sigma h}{\longrightarrow} \Sigma S^{2} \cong S^{3} \stackrel{h}{\longrightarrow} S^{2}
$$

Consider now the inverse images $g^{-1} x$ as $x$ varies over $S^{2}$. If $x \neq x_{ \pm}$then $g^{-1} x:=T_{x}$ in a torus. If $x_{+} \neq x_{-}$, which can be assuned by slightly changing the identification of $\Sigma S^{2}$ with $S^{3}$ if necessary, $g^{-1} x_{+}=T_{+}$and $g^{-1} x_{-}=T_{-}$ are pinched tori. That is a torus with one meridian identified to a point. 


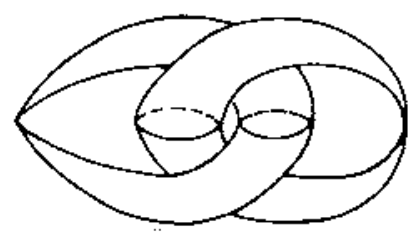

Figure 5

It is interesting to note that if $x_{+}=x_{-}$, i.e. the suspension points both lie in the same fibre of $S^{3}$, then $T_{+}=T_{-}$is a Montesinos twin, that is a pair of spheres meeting in two points. See [Mol and the discussion in [Ma].

Theorem. With the notation above the pinched tori $T_{ \pm} \subset S^{4}$ may be identified with the image of the Fenn-Rolfsen link.

Proof: Generic cross sections of the tori $T_{ \pm}$look as follows: The torus $T_{+}$is indirected by a heavier line than $T_{-}$.

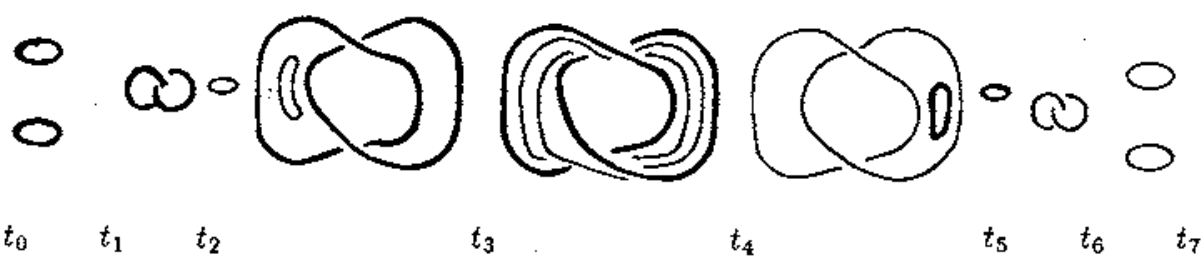

Figure 6. Cross sections of the pinched tori $T_{ \pm}$

In figure 6 critical ocurrences happen at times $t_{0}, \ldots, t_{7}$. They are described in the following list:

$$
\begin{aligned}
& t_{0}-t_{\text {wo }} T_{+} \text {minima } \\
& t_{1}-T_{+} \text {crossing (pinching of a meridian) } \\
& t_{2}-T_{-} \text {minimum } \\
& t_{3}-T_{-} \text {saddle } \\
& t_{4}-T_{+} \text {saddle } \\
& t_{5}-T_{+} \text {maximum } \\
& t_{6}-T_{-} \text {crossing (pinching of a meridian) } \\
& t_{7}-t_{\text {wo }} T_{-} \text {maxima }
\end{aligned}
$$


Figure 6 is shown to represent the same two pinched tori as figure 4 by cancelling a saddle with a minimum on $T_{+}$and a saddle with a maximum on $T_{-}$as illustrated in figure 7 on the component $T_{+}$. This is achieved by pushing over a 3-ball (shown shaded).

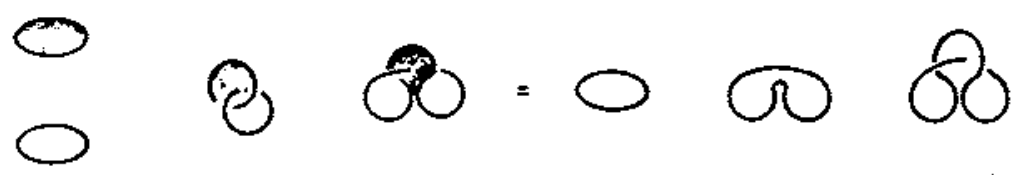

Figure 7. Cancelling a saddle with a minimum

This process applied to $T_{+}$and $T_{-}$results in the cross sections of figure 8 which can be readly seen to be the same as figure 4 .

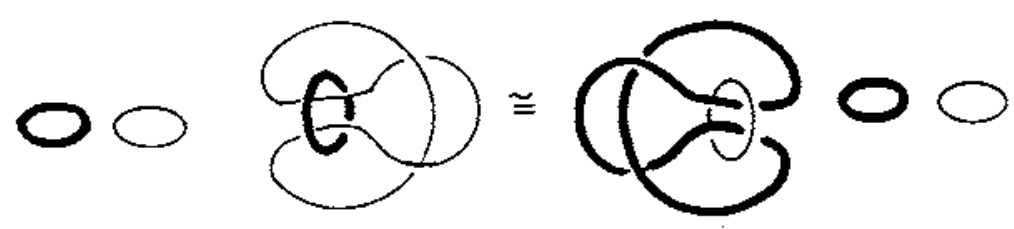

Figure 8

\section{Spanier-Whitehead duality}

The sphere $S^{2}$ has a singular fibration by circles in which $c_{ \pm}=x_{ \pm}$are the two poles and $\left\{c_{\lambda}\right\}$ are the latitude circles, $-1<\lambda<1$.

Consider the following subspaces of $S^{4}$

$$
\begin{gathered}
N_{+}=g^{-1} \bigcup_{[0,1]} c_{\lambda} \quad N_{-}=g^{-1} \bigcup_{[-1,0]} c_{\lambda} \\
M=g^{-1} c_{0}
\end{gathered}
$$

The halfway manifold $M$ is a torus bundle over the circle with bundle map $g \mid M$ and holonomy given by the shear matrix $\left(\begin{array}{ll}1 & 0 \\ \pm & 1\end{array}\right)$.

The manifolds $N_{ \pm}$are regular neighbourhoods of $T_{ \pm}$and $S^{4}=N_{+} \cup N_{-}$, $N_{+} \cap N_{-}=\partial N_{+}=\partial N_{-}=M$.

So $T_{ \pm}$is a deformation retract of $S^{4}-T_{\mp}$ 
Details of the following can be found in [S, W].

Let $A, B$ be spaces with base points $a_{0}, b_{0}$ respectively. Let $A \vee B \subset A \times B$ be the one point union.

$$
A \vee B=A \times b_{0} \cup B \times a_{0}
$$

and let $A \wedge B$ be the smash product

$$
A \wedge B=A \times B / A \vee B
$$

An $n$-duality is a map $u: A \wedge B \rightarrow S^{n}$ which induces an isomorphism between stable homotopy and cohomotopy groups $\pi_{k}^{S} A \rightarrow \pi_{S}^{n-k} B$ under the correspondence

$$
\left\{S^{k} \stackrel{f}{\longrightarrow} A\right\} \longrightarrow\left\{S^{k} \wedge B \stackrel{f \wedge \mathrm{I}}{\longrightarrow} A \wedge B \stackrel{u}{\longrightarrow} S^{n}\right\}
$$

For example if $A, B \subset S^{n+1}$ and $A \cap B=\phi, A$ is a deformation retract of $S^{n+1}-B$ and conversely, then the map $u: A \wedge B \rightarrow S^{n}$ given by

$$
u(a, b):=\frac{a-b}{\|a-b\|}
$$

is an n-duality.

We call the pair $A, B$ in this case an n-duality pair.

It is clear from the discussion above that $T_{+}, T_{-}$is a 3 -duality pair. The map $\varphi$ which defines the $\alpha$-invariant for $f$ factors as follows:

$$
\Psi=\hat{f}_{1} \times \hat{f}_{2}{\stackrel{T}{T_{+}} \wedge T_{-}}_{S^{2} \times S^{2}}^{\longrightarrow}{ }_{\varphi} S^{3}
$$

The map $\Psi$ is of degree 1 and $u$ is a 3 -duality so clearly the class of the composite $[\varphi]=\alpha$ cannot be zero.

\section{References}

[F,H] R.A. FENN AND P.J. HILTON, On links with one codimension 2 component, Preprint.

$[F, R]$ R.A. FENN AND D. ROLFSEN, Spheres may link homotopically in 4-space, J. Lond. Math. Soc. 34 (1986), 177-184.

[K] P. KIRK, Link maps in the four sphere, Differential topology, Lecture notes in Math 1350 (Siegen 1987), 31-43. Springer, 1988

[Ma] Y. Matsumoto, Torus fibrations of 4-dimensional manifolds, Notes. 
[Mo] J. Montesinos, On twins in the four sphere I, Quart. J. Math. Oxford 34 (1983), 171- 199.

[S,W] E.H. SPANIER AND J.H.C. WhITEHEAD, Duality in homotopy theory, Mathematika 2 (1955), 56-80.

\author{
Mathematics Division \\ Sussex University \\ Mathematics and Physics Building \\ Brighton BNI 9QH \\ ENGLAND
}

Rebut el 28 d'Abril de 1989 